\title{
In-situ Observation of Magnetic Skyrmion Crystal Growth from the Conical Phase
}

Tae-Hoon $\mathrm{Kim}^{1}$, Haijun $\mathrm{Zhao}^{2}$, Ben $\mathrm{Xu}^{3}$, Brandt Jensen ${ }^{1}$, Alexander King ${ }^{1}$, Matthew Kramer ${ }^{1}$, Liqin $\mathrm{Ke}^{1}$ and Lin Zhou ${ }^{1}$

${ }^{1}$ Ames Laboratory, Ames, Iowa, United States, ${ }^{2}$ Southeast University, Nanjing, Jiangsu, China (People's Republic), ${ }^{3}$ Tsinghua University, Beijing, Beijing, China (People's Republic)

Magnetic skyrmion is a nanoscale vortex-like spin structure that have attracted considerable interests as information carriers for future spintronic devices. As a quasiparticle, skyrmions in chiral magnets can form SkX lattices analogous to crystallization in molecular systems. This quasiparticle crystal introduces an opportunity to explore intriguing phenomenon that is not expected in growth of crystals made of real particles. In spite of extensive previous research on skyrmion imaging and formation mechanisms [1], real-space observation of $\mathrm{SkX}$ growth is still challenging due to its sensitivity to temperature and magnetic field [2]. In this study, we focus on using in-situ Lorentz transmission electron microscopy (LTEM) to study SkX growth mechanism and behavior.

A Co8Zn10 Mn2 (110) thin plate was fabricated using focused ion beam system. The plate was approximately $200 \mathrm{~nm}$ thick. In-situ LTEM observation was conducted on an FEI Titan Themis by using FEI NanoEx-Tm-i/v in-situ TEM holder which enabled rapid heating and precise temperature control. The external magnetic field was applied along the electron beam direction by partially exciting the objective lens. LTEM videos were taken with $50 \mathrm{~ms}$ of temporal resolution by FEI Ceta camera to record SkX growth.

Figure 1 shows the nucleation of skyrmion and $\mathrm{SkX}$ revealed by in-situ observation. The helical phase transformed into the conical phase upon reaching the targeted experimental condition (Fig. 1a, b). A skyrmion embryo appeared and developed into a full contrast skyrmion (Fig. 1c). The first skyrmion acted as a preferred nucleation site for subsequent skyrmions (Fig. d-e). Skyrmion obligomers grew to a cluster (Fig. 1f-h) by monomer-by-monomer addition (MA) of skyrmions. The 3D view of magnetic structure of MA mechanism is shown in Fig. 1i.

Figure 2 represents self-splitting (SS) mechanism of skyrmion growth at defects inside the SkX beside MA process. A skyrmion with seven neighbors (Fig. 2a) rapidly divided into two skyrmions (Fig. 2b). The imperfect skyrmion coordination with adjacent fivefold and sevenfold-coordinated skyrmions was evolved by SS mechanism that is not possible in a crystal made of real molecules. Micromagnetic simulation revealed that SS starts at the surface of the sample by creating a magnetic monopole (Fig. 3c). The monopole then moves toward the opposite surface, and unzip the skyrmion into two. Studies on kinetics of skyrmion formation is in progress and will be discussed as well $[3,4]$. 

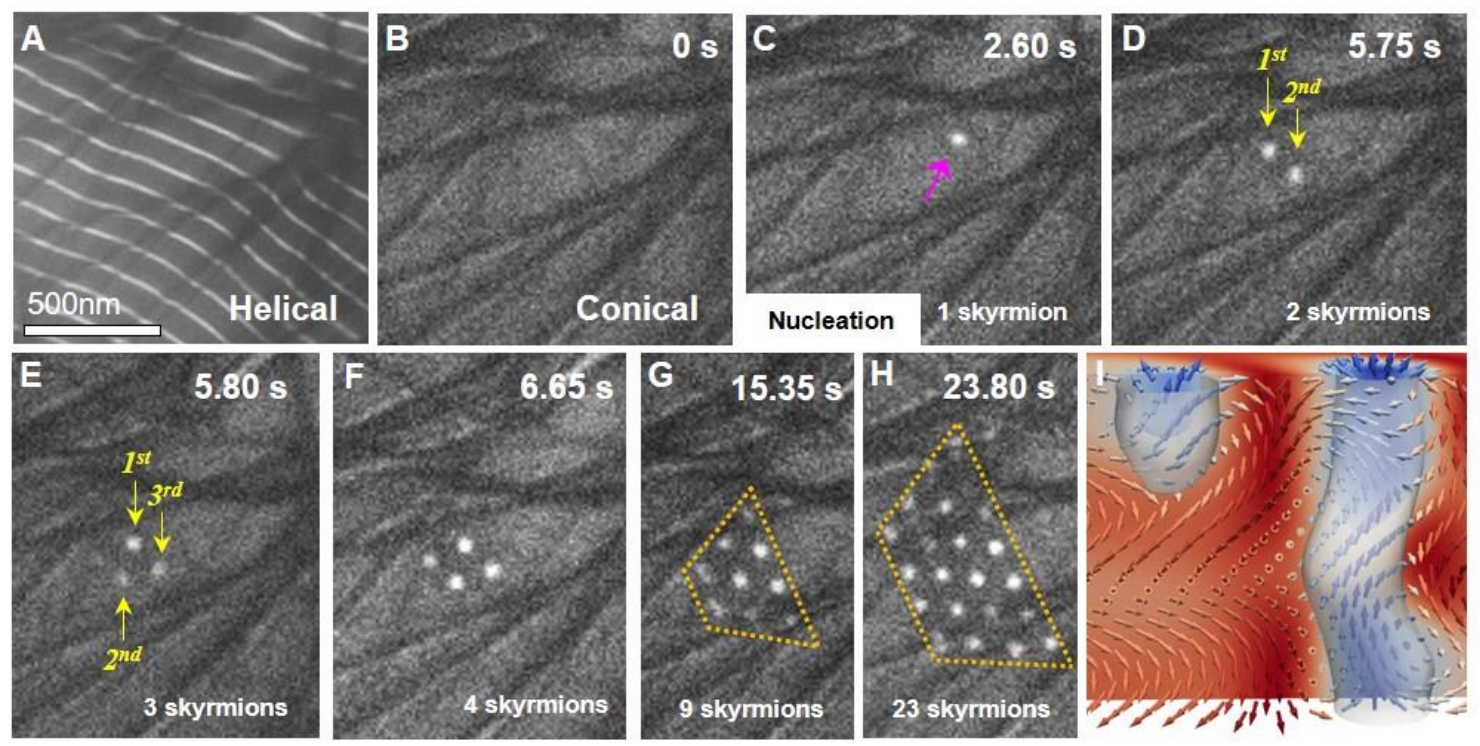

Figure 1. Skyrmion and SkX nucleation. (a) Helical phase with periodicity of $\sim 115 \mathrm{~nm}$ at room temperature under $0 \mathrm{mT}$. (b-h) Sequence of LTEM images after sample reached $84{ }^{\circ} \mathrm{C}$ under $135 \mathrm{mT}$. (b) Conical phase. (c) Nucleation of the first skyrmion. (d-h) Subsequent SkX growth through MA mechanism. (i) 3D view of magnetic structure of MA mechanism.
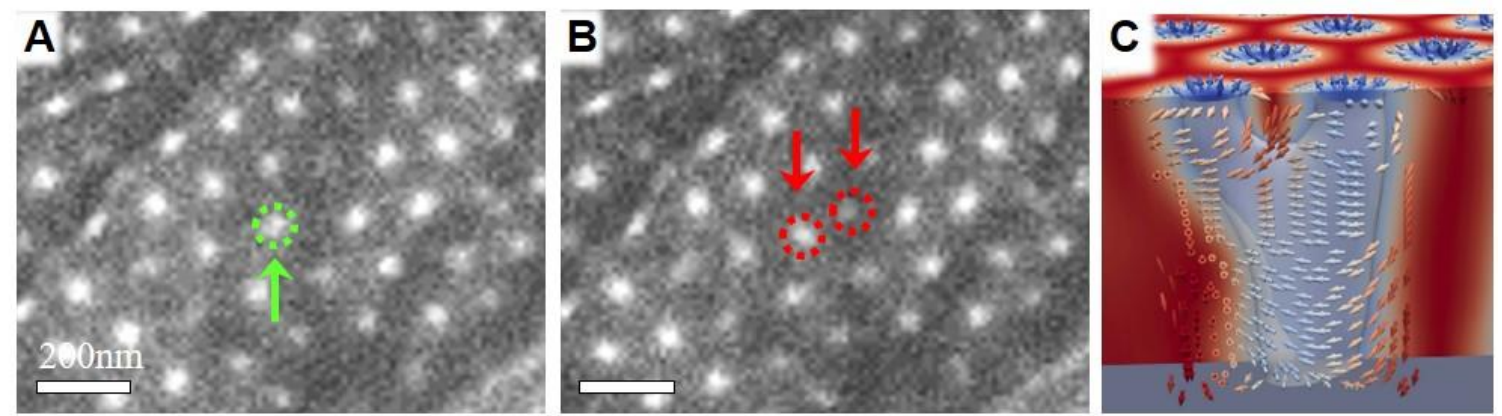

Figure 2. Self-splitting (SS) mechanism of SkX growth. (a) The green circle shows the center skyrmion of sevenfold disclination. (b) Splitting of the center skyrmion into two skyrmions (red). (c) 3D view of magnetic structure of skyrmion SS process.

\section{References}

[1] A O Leonov et al, Phys. Rev. B 98, (2018) 1-9.

[2] Y Tokunaga et al, Nat. Commun 6, (2015) 7638.

[3] T Kim et al, arXiv Prepr. arXiv1912.03226 (2019)

[4] This work was supported in part by the Laboratory Directed Research and Development funds through Ames Laboratory. All TEM-related work was performed using instruments in the Sensitive Instrument Facility in Ames Laboratory. Ames Laboratory is operated for the U.S. Department of Energy by Iowa State University under Contract No. DE-AC02-07CH11358. 